\title{
Efficient Importance Sampling for ML Estimation of SCD Models
}

L. Bauwens and F. Galli

Discussion Paper 2007-32

Département des Sciences Économiques

de l'Université catholique de Louvain 


\title{
CORE DISCUSSION PAPER
}

$2007 / 53$

\section{EFFICIENT IMPORTANCE SAMPLING \\ FOR ML ESTIMATION OF SCD MODELS}

\author{
Luc Bauwens ${ }^{1}$ and Fausto Galli ${ }^{2}$
}

August 17, 2007

\begin{abstract}
The evaluation of the likelihood function of the stochastic conditional duration model requires to compute an integral that has the dimension of the sample size. We apply the efficient importance sampling method for computing this integral. We compare EIS-based ML estimation with QML estimation based on the Kalman filter. We find that EIS-ML estimation is more precise statistically, at a cost of an acceptable loss of quickness of computations. We illustrate this with simulated and real data. We show also that the EIS-ML method is easy to apply to extensions of the SCD model.
\end{abstract}

Keywords: stochastic conditional duration, importance sampling.

JEL Classification: C13, C15, C41

\footnotetext{
${ }^{1}$ CORE and Department of Economics, Université catholique de Louvain.

${ }^{2}$ Swiss Finance Institute at University of Lugano

Bauwens's work was supported by a FSR grant from UCL. He is also a member of ECORE, a newly created association between ECARES and CORE.

This text presents research results of the Belgian Program on Interuniversity Poles of Attraction initiated by the Belgian State, Prime Minister's Office, Science Policy Programming. The scientific responsibility is assumed by the authors.
} 


\section{Introduction}

The stochastic conditional duration (SCD) model is a dynamic duration model that was proposed by Bauwens and Veredas (2004) as an alternative to the autoregressive conditional duration model of Engle and Russell (1998). These models are typically applied to analyze the dynamics of the intra-daily trading activity of financial markets. The SCD model uses two unobservable stochastic components for one observable variable (the duration), implying that one of the stochastic terms must be integrated over the whole sample to compute the likelihood function. The fact that the variables to be integrated enter the model nonlinearly leads to the necessity to compute this high-dimensional integral by simulation. The same problem arises for the stochastic volatility (SV) model, since it has the same structure as the SCD model.

The solution proposed by Bauwens and Veredas in their original paper circumvented the integration problem since they used quasi-maximum likelihood (QML) estimation based on an approximation of the model by a linear space state representation. This makes it possible to use the Kalman filter to approximate the likelihood function. This method has the advantage of being simple and fast in terms of numerical computation and of providing consistent and asymptotically normal estimators (under suitable regularity conditions), but it is in principle suboptimal in finite samples.

To avoid approximations, in the literature on SV models other estimation procedures have been proposed, for instance the generalized method of moments (GMM), the efficient method of moments (EMM), and Bayesian estimation based on Markov chain Monte Carlo (MCMC) sampling. For a survey of these procedures see E. Ghysels and Renault (1996). Some of these methods have been extended to the estimation of SCD models. Ning (2004) proposes to estimate SCD models via the empirical characteristic function (ECF) and GMM. Maximum likelihood estimation based on MCMC integration of the latent variables is instead adopted by Feng, Jiang, and Song (2004) to estimate a SCD model in the form proposed by Bauwens and Veredas (2004) and an extended version with a leverage effect determined by the presence of past durations in the mean of the latent variable. MCMC is used also by C. Strickland 
and Martin (2006) in the context of Bayesian estimation.

A relatively new method for computing the integral needed for evaluating the likelihood function of models with latent variables relies on the efficient importance sampling (EIS) procedure, recently developed by Richard and Zhang (1998). This method consists in an extension of the well known importance sampling technique and seems to be particularly well suited for the computation of the multidimensional though relatively well behaved integral needed for evaluation the SCD likelihood. An application to an extended family of SV models is provided in Liesenfeld and Richard (2003) who illustrate the flexibility of this algorithm.

The purpose of this article is to extend to the original SCD model of Bauwens and Veredas (2004) the EIS method of numerical sampling, to compare it to the QML estimation method on simulated and real data, and to use it in order to analyze some extensions of the original specification proposed by Bauwens and Veredas. Our main finding is that for the order of magnitude of the sample sizes used in practice, i.e. a few thousands, there is a substantial efficiency gain in favour of EIS-ML.

The article is organized in the following way. In section 2 we present the main features of the SCD model which are necessary to the subsequent analysis. In Section 3 we detail the EIS numerical integration method employed for ML estimation of the SCD model. In section 4 we apply this method to several simulated data sets and compare the sampling distributions of EIS-ML and QML estimates. In section 5 the EIS-ML technique is applied to the same dataset used in Bauwens and Veredas (2004) and a comparison is drawn. Two examples of parametric extensions of the SCD model which are easily estimated by EIS-ML are provided in Section 6. The last section contains our concluding remarks.

\section{SCD models: main features}

In this section, we present briefly the SCD model. A more detailed description can be found in Bauwens and Veredas (2004). If we denote by $x_{i}$ the duration between two events that happened at times $t_{i-1}$ and $t_{i}$, and assume that the stochastic process $\left\{x_{i}\right\}$ generating the durations is doubly infinite ( $i$ goes from $-\infty$ to $+\infty$ ), the stochastic conditional duration 
model can be written as

$$
x_{i}=e^{\psi_{i}} \epsilon_{i}
$$

with

$$
\psi_{i}=\omega+\beta \psi_{i-1}+u_{i}
$$

where $|\beta|<1$ to ensure the stationarity of the process, and

$$
\begin{aligned}
u_{i} & \sim i i d \mathcal{N}\left(0, \sigma^{2}\right), \\
\epsilon_{i} & \sim i i d p\left(\epsilon_{i}\right),
\end{aligned}
$$

with $p($.$) a distribution with positive support, and u_{j}$ independent of $\epsilon_{i}, \forall i, j$.

The moments and autocorrelation function of this process are

$$
\begin{aligned}
& \mathrm{E}\left(x_{i}\right)=\mu e^{\frac{\omega}{1-\beta}+\frac{1}{2} \frac{\sigma^{2}}{1-\beta^{2}}} \\
& 1+\delta_{x}^{2}=\left(1+\delta^{2}\right) e^{\frac{\sigma^{2}}{1-\beta^{2}}} \\
& \rho_{k}=\frac{e^{\frac{\sigma^{2} \beta^{k}}{1-\beta^{2}}-1}}{\left(1+\delta^{2}\right)\left(e^{\frac{\sigma^{2}}{1-\beta^{2}}}-1\right)} \approx \frac{\sigma^{2} \beta^{k} /\left(1-\beta^{2}\right)}{\left(1+\delta^{2}\right)\left(e^{\frac{\sigma^{2}}{1-\beta^{2}}}-1\right)} \approx \beta \rho_{k-1},
\end{aligned}
$$

where $\mu$ stands for $\mathrm{E}\left(\epsilon_{i}\right), \delta_{x}$ for the dispersion index (i.e. the standard deviation to mean ratio) of $x_{i}$, and $\delta$ for the dispersion index of $\epsilon_{i}$. The autocorrelation function $\rho_{k}$ geometrically decreases at rate $\beta$ only asymptotically with respect to $k$, while for small $k$ the decrease rate is smaller.

Given a sequence $x$ of $n$ realizations of the process, with density $g\left(x \mid \psi, \theta_{1}\right)$ indexed by the parameter vector $\theta_{1}$, conditional on a vector $\psi$ of latent variables of the same dimension as $x$, and given the density $h\left(\psi \mid \theta_{2}\right)$ indexed by the parameter $\theta_{2}$, the likelihood function of $x$ can be written as:

$$
L(\theta ; x)=L\left(\theta_{1}, \theta_{2} ; x\right)=\int g\left(x \mid \psi, \theta_{1}\right) h\left(\psi \mid \theta_{2}\right) d \psi
$$

Actually, the integrand in the previous equation is the joint density $f\left(x, \psi \mid \theta_{1}, \theta_{2}\right)$. Given the 
assumptions we made, it can be sequentially decomposed as

$$
f\left(x, \psi \mid \theta_{1}, \theta_{2}\right)=\prod_{i=1}^{n} p\left(x_{i} \mid \psi_{i}, \theta_{1}\right) q\left(\psi_{i} \mid \psi_{i-1}, \theta_{2}\right)
$$

where $p\left(x_{i} \mid \psi_{i}, \theta_{1}\right)$ is obtained from $p\left(\epsilon_{i}\right)$ using the change of variable in (1) (so that $\theta_{1}$ corresponds to the parameters of $p()$.$) , and q\left(\psi_{i} \mid \psi_{i-1}, \theta_{2}\right)$ is the Gaussian density $\mathcal{N}\left(\omega+\beta \psi_{i-1}, \sigma^{2}\right)$ (so that $\theta_{2}$ includes $\omega, \beta$ and $\sigma^{2}$ ). Given the functional form usually adopted for $p\left(\epsilon_{i}\right)$ (Weibull, gamma, generalized gamma...), the multidimensional integral in (6) cannot be solved analytically and must be computed numerically by simulation.

To perform a QML estimation, one can use the following transformation of the model:

$$
\begin{aligned}
& \ln x_{i}=\eta+\psi_{i}+\xi_{i}, \\
& \psi_{i}=\omega+\beta \psi_{i-1}+u_{i},
\end{aligned}
$$

where $\xi_{i}=\ln \epsilon_{i}-\eta$ and $\eta=\mathrm{E}\left(\ln \epsilon_{i}\right)$. This puts the model in state space form with zero mean errors. The ensuing distribution of $\xi_{i}$ can be approximated by a Gaussian one, and the Kalman filter applied to compute the approximate likelihood.

\section{EIS based ML estimation}

Alternative approaches to QML inference for the SCD model can be based on Monte Carlo methods. These methods are widely used in Bayesian inference to evaluate moments of highdimensional posterior densities when they are not known analytically. One reason for their success is the relative ease with which they can be applied. EIS, in particular, allows a very accurate evaluation of the likelihood function and has been shown to be quite reliable for the estimation of SV models and of latent factor intensity models, see Bauwens and Hautsch (2006). Another advantage of this algorithm is that its basic structure does not depend on a specific model. This renders changes in the distributional assumptions for the underlying random variables rather simple.

For a detailed presentation of the algorithm, we refer the reader to Richard and Zhang 
(1998). In this section, we present the basics of its motivation and functioning and we detail its implementation in the context of the ML estimation of SCD models.

Assume one has to evaluate a unidimensional functional integral of the form

$$
G(y)=\int_{\Lambda} g(y, \lambda) p(\lambda) d \lambda
$$

where $g$ is a integrable function with respect to a density $p(\lambda)$ with support $\Lambda$. The vector $y$ denotes an observed data vector, which in our context corresponds to the observed durations.

A Monte Carlo (MC) estimate of (8) is

$$
\bar{G}_{S}(y)=\frac{1}{S} \sum_{i=1}^{S} g\left(y, \tilde{\lambda}_{i}\right),
$$

where the $\tilde{\lambda}_{i}$ 's are draws from $p$ and $S$ is the number of draws. In cases where one cannot generate directly draws from $p(\lambda)$, one can resort to importance sampling (IS). The IS principle consists of replacing the initial sampler $p(\lambda)$ with an auxiliary parametric importance sampler $m(\lambda, a)$, which is an easy-to-simulate density for $\lambda$, indexed by the parameter vector a. To apply importance sampling, equation (8) is transformed into

$$
G(y)=\int_{\Lambda} g(y, \lambda) \frac{p(\lambda)}{m(\lambda, a)} m(\lambda, a) d \lambda
$$

and the corresponding IS-MC estimate of $G(y)$ is

$$
\bar{G}_{S, m}(y, a)=\frac{1}{S} \sum_{i=1}^{S} g\left(y, \tilde{\lambda}_{i}\right) \frac{p\left(\tilde{\lambda}_{i}\right)}{m\left(\tilde{\lambda}_{i}, a\right)},
$$

where the $\tilde{\lambda}_{i}$ 's now denote draws from the IS density $m$.

The aim of efficient importance sampling (EIS) is to minimize the MC variance of the estimator in (11) by selecting optimally the parameters $a$ of the importance function density $m$ given a functional form for $m$ (for instance the Gaussian density). 
Given independent draws $\tilde{\lambda}_{i}$ 's, the sampling variance of $\bar{G}_{S, m}(y, a)$ is given by

$$
\operatorname{Var}\left(\bar{G}_{S, m}(y, a)\right)=G(y) V(a, y)
$$

where

$$
V(a, y)=\frac{1}{G(y)} \int_{\Lambda}\left[\frac{g(y, \lambda) p(\lambda)}{m(\lambda, a)}-G(y)\right]^{2} m(\lambda, a) d \lambda
$$

If we denote by $k(\lambda, a)$ the density kernel of the IS sampler $m(\lambda, a)$ and by $\chi(a)$ its integral, such that $m(y, a)=\frac{k(\lambda, a)}{\chi(a)}, V(a, y)$ in 13 can be rewritten as

$$
V(a, y)=\int_{\Lambda} h\left(d^{2}(y, a, \lambda)\right) g(y, \lambda) p(\lambda) d \lambda,
$$

where

$$
d(y, a, \lambda)=\ln g(y, \lambda)-\ln p(\lambda)-\ln k(\lambda, a)-\ln G(y)-\ln \chi(a),
$$

and

$$
h(c)=e^{\sqrt{c}}+e^{-\sqrt{c}}-2=2 \sum_{i=1}^{\infty} \frac{c^{i}}{(2 i) !} .
$$

Noting that the term $d(y, a, \lambda)$ is supposed to be small if an efficient sampler is used, the function $h(c)$ can be approximated by its leading term $c$ to get

$$
V(a, y) \approx \tilde{V}(a, y)=\int_{\Lambda} d^{2}(y, a, \lambda) g(y, \lambda) p(\lambda) d \lambda
$$

Minimizing the MC variance amounts then to minimizing the quadratic term $d^{2}(y, a, \lambda)$. It can be shown that if the importance sampler $m$ belongs to the exponential family, the problem remarkably simplifies to a least squares minimization problem for the components of the vector of auxiliary parameters $a$.

Extending the EIS approach to the case where $\lambda$ is of high dimension, like in the SCD model (where $\lambda$ corresponds to the $\psi$ vector), requires that we can decompose the minimization problem in a series of unidimensional subproblems.

A natural MC estimate of the likelihood function in (7), which is of the type of (9), is 
given by

$$
\tilde{L}(\theta ; x)=\frac{1}{S} \sum_{j=1}^{S}\left[\prod_{i=1}^{n} p\left(x_{i} \mid \tilde{\psi}_{i}^{(j)}, \theta_{1}\right)\right]
$$

where $\tilde{\psi}_{i}^{(j)}$ denotes a draw from the density $q\left(\psi_{i} \mid \psi_{i-1}, \theta_{2}\right)$. This approach bases itself only on the information provided by the distributional assumptions of the model and does not consider the information that comes from the observed sample. It turns out that this estimator is highly inefficient since its sampling variance rapidly increases with the sample size. In any practical case of a duration data set, where the sample size $n$ lies between 500 and 50000 observations, the Monte Carlo sampling size $S$ required to give precise enough estimates of $L(\theta ; x)$ would be too high to be affordable and it turns out that this estimator cannot be relied on practically.

EIS tries to make use of the information provided by the observed data in order to come to a reasonably fast and reliable numerical approximation. Let $\left\{m\left(\psi_{i} \mid \psi_{i-1}, a_{i}\right)\right\}_{i=1}^{n}$ be a sequence of auxiliary samplers indexed by the set of auxiliary parameter vectors $\left\{a_{i}\right\}_{i=1}^{n}$. These densities can be defined as a parametric extension of the natural samplers $\left\{q\left(\psi_{i} \mid \psi_{i-1}, \theta_{2}\right)\right\}_{i=1}^{n}$. We rewrite the likelihood function as

$$
L(\theta ; x)=\int\left[\prod_{i=1}^{n} \frac{f\left(x_{i}, \psi_{i} \mid x_{i-1}, \psi_{i-1}, \theta\right)}{m\left(\psi_{i} \mid \psi_{i-1}, a_{i}\right)} \prod_{i=1}^{n} m\left(\psi_{i} \mid \psi_{i-1}, a_{i}\right)\right]
$$

Then, its corresponding IS-MC estimator (the equivalent of (11)) is given by

$$
\tilde{L}(\theta ; x, a)=\frac{1}{S} \sum_{j=1}^{S}\left[\prod_{i=1}^{n} \frac{f\left(x_{i}, \tilde{\psi}_{i}^{(j)}\left(a_{i}\right) \mid x_{i-1}, \tilde{\psi}_{i-1}^{(j)}\left(a_{i-1}\right)\right)}{m\left(\tilde{\psi}_{i}^{(j)}\left(a_{i}\right) \mid \tilde{\psi}_{i-1}^{(j)}\left(a_{i-1}\right), a_{i}\right)}\right]
$$

where $\left\{\left(\tilde{\psi}_{i}^{(j)}\left(a_{i}\right)\right\}_{i=1}^{n}\right.$ are trajectories drawn from the auxiliary samplers.

Relying on the factorized expression of the likelihood, the MC variance minimization problem can be decomposed in a sequence of subproblems for each element $i$ of the sequence of observations, provided that the elements depending on the lagged values $\psi_{i-1}$ are transferred back to the $(i-1)$-th minimization subproblem. More precisely, if we decompose $m$ in the 
product of a function of $\psi_{i}$ and $\psi_{i-1}$ and one of $\psi_{i-1}$ only, such that

$$
m\left(\psi_{i} \mid \psi_{i-1}, a_{i}\right)=\frac{k\left(\psi_{i}, a_{i}\right)}{\chi\left(\psi_{i-1}, a_{i}\right)}=\frac{k\left(\psi_{i}, a_{i}\right)}{\int k\left(\psi_{i}, a_{i}\right) d \psi_{i}},
$$

we can set up the following minimization problem:

$$
\hat{a}_{i}(\theta)=\arg \min _{a_{i}} \sum_{j=1}^{M}\left\{\ln \left[f\left(x_{i}, \tilde{\psi}_{i}^{(j)} \mid \tilde{\psi}_{i-1}^{(j)}, x_{i-1}, \theta\right) \chi\left(\tilde{\psi}_{i}^{(j)}, \hat{a}_{i+1}\right)\right]-c_{i}-\ln \left(k\left(\tilde{\psi}_{i}^{(j)}, a_{i}\right)\right\}^{2},\right.
$$

where $c_{i}$ is constant that must be estimated along with $a_{i}$. If the density kernel $k\left(\psi_{i}, a_{i}\right)$ belongs to the exponential family of distributions, the problem becomes linear in $a_{i}$, and this greatly improves the speed of the algorithm, as a least squares formula can be employed instead of an iterative routine.

The estimated $\hat{a}_{i}$ are then substituted in (20) to obtain the EIS estimate of the likelihood. The EIS algorithm can be initialized by direct sampling, as in equation (18), to obtain a first series of $\tilde{\psi}_{i}^{(j)}$ and then iterated to allow the convergence of the sequences of $\left\{a_{i}\right\}$, which is usually obtained after 3 to 5 iterations. EIS-ML estimates are finally obtained by maximizing $\tilde{L}(\theta ; x, a)$ with respect to $\theta$.

If we adopt a Weibull distribution for $\epsilon_{i}$ with parameter $\gamma\left(=\theta_{1}\right)$ and a $N\left(0, \sigma^{2}\right)$ one for $u_{i}$, we come up with the following expressions:

$$
\begin{aligned}
p\left(x_{i} \mid \psi_{i-1}, \gamma\right) & =\frac{\gamma}{e^{\psi_{i-1}}}\left(\frac{x_{i}}{e^{\psi_{i-1}}}\right)^{\gamma-1} \exp \left\{-\left(\frac{x_{i}}{e^{\psi_{i-1}}}\right)^{\gamma}\right\}, \\
q\left(\psi_{i} \mid \psi_{i-1}, \theta_{2}\right) & =\frac{1}{\sigma \sqrt{2 \pi}} \exp \left\{-\frac{1}{2 \sigma^{2}}\left(\psi_{i}-\omega-\beta \psi_{i-1}\right)^{2}\right\} .
\end{aligned}
$$

A convenient choice for the auxiliary sampler $m\left(\psi_{i}, a_{i}\right)$ is a parametric extension of the natural sampler $q\left(\psi_{i} \mid \psi_{i-1}, \theta_{2}\right)$, in order to obtain a good approximation of the integrand without too heavy a cost in terms of analytical complexity. Following Liesenfeld and Richard (2003), we can start by the following specification of the function $k\left(\psi_{i}, a_{i}\right)$ :

$$
k\left(\psi_{i}, a_{i}\right)=q\left(\psi_{i} \mid \psi_{i-1}, \theta_{2}\right) \zeta\left(\psi_{i}, a_{i}\right)
$$


where $\zeta\left(\psi_{i}, a_{i}\right)=\exp \left\{a_{1, i} \psi_{i}+a_{2, i} \psi_{i}^{2}\right\}$ and $a_{i}=\left(a_{1, i} a_{2, i}\right)$. This specification is rather straightforward and has two advantages. Firstly, as $q\left(\psi_{i} \mid \psi_{i-1}, \theta_{2}\right)$ is present in a multiplicative form, it cancels out in the objective function in $(22)$, which becomes a least squares problem with $\ln \zeta\left(\psi_{i}, a_{i}\right)$ that serves to approximate $\ln p\left(x_{i} \mid \psi_{i-1}, \theta_{1}\right)+\ln \chi\left(\psi_{i-1}, a_{i}\right)$. Secondly, such a functional form for $k$ leads to a distribution of the auxiliary sampler $m\left(\psi_{i}, a_{i}\right)$ that remains Gaussian, as stated in the following theorem, whose proof is given in the appendix.

Theorem 1 If the functional forms for $q\left(\psi_{i} \mid \psi_{i-1}, \theta_{2}\right)$ and $k\left(\psi_{i}, a_{i}\right)$ are as in equations (24) and 25) respectively, then the auxiliary density $m\left(\psi_{i} \mid \psi_{i-1}, a_{i}\right)=\frac{k\left(\psi_{i}, a_{i}\right)}{\chi\left(\psi_{i-1}, a_{i}\right)}$ is Gaussian, with conditional mean and variance given by:

$$
\begin{aligned}
& \mu_{i}=v_{i}^{2}\left(\frac{\omega+\beta \psi_{i-1}}{\sigma^{2}}+a_{1, i}\right), \\
& v_{i}^{2}=\frac{\sigma^{2}}{1-2 \sigma^{2} a_{2, i}},
\end{aligned}
$$

and the function $\chi\left(\psi_{i-1}, a_{i}\right)$ is given by

$$
\frac{1}{\sqrt{1-2 \sigma^{2} a_{2, i}}} \exp \left\{\frac{\sigma^{2}}{2\left(1-2 \sigma^{2} a_{2, i}\right)}\left(\frac{\omega+\beta \psi_{i-1}}{\sigma^{2}}+a_{1, i}\right)^{2}-\frac{1}{2}\left(\frac{\omega+\beta \psi_{i-1}}{\sigma}\right)^{2}\right\}
$$

By applying these results, it is possible to compute the likelihood function of the SCD model for a given value of $\theta$, based upon the following steps:

Step 1. Use the natural sampler $q\left(\psi_{i} \mid \psi_{i-1}, \theta_{2}\right)$ to draw $S$ trajectories of the latent variable $\left\{\tilde{\psi}_{i}^{(j)}\right\}_{i=1}^{n}$, as in 18 .

Step 2. The draws obtained in step 1 are used to solve for each $i$ (in the order from $n$ to 1 ) the least squares problems described in $(22)$, which takes the form of the auxiliary linear regression:

$$
\begin{array}{ll}
\ln \gamma-\gamma \tilde{\psi}_{i}^{(j)}+(\gamma-1) \ln x_{i}-\left(\frac{x_{i}}{e^{\tilde{\psi}_{i}^{(j)}}}\right)^{\gamma}+\ln \chi\left(\tilde{\psi}_{i}^{(j)}, \hat{a}_{i+1}\right)= & \\
a_{0, i}+a_{1, i} \tilde{\psi}_{i}^{(j)}+a_{2, i}\left(\tilde{\psi}_{i}^{(j)}\right)^{2}+\varepsilon_{i}^{(i)}, & j=1, \ldots, S,
\end{array}
$$


where $\varepsilon_{i}^{(i)}$ is the error term, $a_{0, i}$ is a constant term, and $\chi\left(\tilde{\psi}_{i}^{(j)}, \hat{a}_{i+1}\right)$ is set equal to 1 for $i=n$ and defined by (27) for $i<n$.

Step 3. Use the estimated auxiliary parameters $\hat{a}_{i}$ to obtain $S$ trajectories $\left\{\tilde{\psi}_{i}^{(j)}\left(\hat{a}_{i}\right)\right\}_{i=1}^{N}$ from the auxiliary sampler $m\left(\psi_{i} \mid \psi_{i-1}, \hat{a}_{i}\right)$, applying the result of theorem 1 .

Step 4. Return to step 2, this time using the draws obtained with the auxiliary sampler. Steps 2, 3 and 4 are usually iterated a small number of times (from 3 to 5), until a reasonable convergence of the parameters $\hat{a}_{i}$ is obtained.

Once the auxiliary trajectories have attained a reasonable degree of convergence, the simulated samples can be plugged in formula 20 to obtain an EIS estimate of the likelihood. This procedure is embedded in a numerical maximization algorithm that converges to a maximum of the likelihood function. After convergence, we compute the standard errors from the Hessian matrix.

\section{Simulation results}

In order to assess the gain in performance allowed by EIS-ML estimation in comparison with QML, we conducted several repeated simulation experiments with different parameter configurations. As the QML estimator should be consistent but inefficient in relatively small samples, trajectories of 250, 500, 1000, 5000 and 10000 observations form a SCD data generating process (DGP) were simulated 1000 times and the model was estimated both by EIS-ML and by QML. The idea to use as much as 10000 observations comes from the wish to judge the loss of efficiency of QML relative to EIS-ML. Moreover, such sample sizes are far from unusual for real data sets of durations.

The estimations were performed using the MaxSQPF constrained maximization function of Ox console 3.40, under Windows XP with a dual core Intel 2.0 Gb processor. The speed of estimation varies from a few seconds for a series of 250 data to a few minutes for a 10000 data one. EIS -ML estimation is much slower, with a computing time ranging from 10 to 100 times the one required by QML with the Kalman filter. This should not come as a surprise 
and we suspect that alternative estimation strategies, such as Bayesian MCMC, would be even slower than EIS-ML, as the results of Bauwens and Rombouts (2004) for the SV model clearly show.

The DGP is defined by equations (1)-(4), plus formula (23). The parameter values used in the simulations of the DGP were the following:

- $\omega=0.0$

- $\beta=0.9$,

- $\sigma=0.05$ and 0.2 ,

- $\gamma=0.8$ and 1.1,

thus leading to four combinations. The starting values for likelihood optimizations were set for all estimations to $\omega=0.0, \beta=0.85, \sigma=0.15$ and $\gamma=1.05$, but we checked that other reasonable starting values provided quite similar results to those discussed below.

Tables 1 to 5 contain the means, standard deviations and mean-squared errors of the 1000 estimates for each experiment, and figures 1 to 4 display the corresponding sampling densities (obtained by kernel based smoothing). As a first remark, it can be noticed that in both estimation methods there is a tendency to underestimate the autoregressive parameter $\beta$ and to overestimate the parameter $\sigma$, especially when the latter takes the low value of 0.05. Anyway, also in these cases the EIS-ML method provides estimates which in mean are closer to the DGP parameters than the QML one. The most striking result concerns the efficiency of the estimators: the EIS-ML estimated standard deviations of the estimates are always remarkably smaller than the QML ones, in particular when the parameter $\sigma$ is equal to 0.05 . The combination of smaller bias and variance is reflected clearly in the mean-squared errors, which are sensibly lower across the board for the EIS-ML method. The better general performance of the EIS-ML estimator can be appreciated also by a visual inspection of the sampling densities.

Looking at the tables and at figures 2 and 3 it is easy to remark how poor the performance of QML estimator is when the parameter $\sigma$ is small. To better illustrate the issue, Figure 5 
shows for each parameter the graph of the estimated standard deviations against the value of $\sigma$ in the DGP, for a sample size of 1000. These results are based on additional simulations (for values of $\sigma$ ranging from 0.02 to 0.7 ). This figure shows that for small values of $\sigma$ in the DGP, both estimation methods tend to be imprecise. This is understandable, since as $\sigma$ tends to 0 , the parameter $\beta$ becomes unidentified, so that the likelihood function becomes flat. We also see in figures 2 and 3 that for the sampling distribution of the estimates of $\beta$ have a mode at (or close to) zero. However, the problem of is far more pronounced for QML than for EIS-ML.

In order to dispel the doubt that the QML estimator might not be consistent (or that the computer program is poorly written) we present in table 6 the results based on ten QML estimations with one million data. From all this evidence we conclude that when the sample size is not very large (of the order of hundreds of thousands), QML estimation can be extremely inefficient if the standard deviation of second error term is small.

\section{Estimation results for real data}

In this section, we apply EIS-ML estimation to some data sets used by Bauwens and Veredas (2004) for QML estimation, and we compare the results. The SCD model is exactly the same as in the previous section, and as in these authors' article.

The considered data sets correspond to five stocks traded at the New Your Stock Exchange (NYSE): Boeing, Coca Cola, Disney, Exxon and Ibm (the last one was not used by Bauwens and Veredas). The data were extracted from the trades and quotes database (TAQ) of the NYSE and are relative to the months of September, October and November, 1996. From the original trade and quote durations, price and volume duration were computed. Price durations measure the amount of time before observing a given cumulated variation (up or down) of the price (in this case, $\$ 1 / 8$ ). Analogously, volume durations measure the amount of time necessary to observe a cumulative traded volume of a given amount (25000 shares). As it is customary in the literature on financial durations, the durations have been purged of their seasonal component. An important feature of the trade and quote data is in fact 
the strong seasonality featured, both on a daily and a weekly basis, by key characteristics of the duration processes. Price and volume durations feature a strong intra-day effect, being smaller at the start and at the end of the trading day than around lunch time. Moreover, this effect may depend on the day of the week. These deterministic time-of-day and day-of-the week effect are controlled by nonparametrically regressing the observed durations of each day of the week on the time of the day, and by defining the deseasonalized durations as the original ones divided by the fitted values of the regression.

In tables 7 and 8 we present the estimated parameters of the QML and EIS-ML estimations. For volume duration, even if the values taken by the estimates of the parameters for the two methods considered are substantially the same, it is noticeable that the standard errors of EIS-QML are generally lower, sometimes substantially, than the QML ones. We also report in the table the computed dispersion index $\left(\tilde{\delta}_{x}\right)$ of the durations implied by the estimates. This is computed by plugging in formula (5) the point estimates. We see that the data dispersion index is better approximated if we estimate with the EIS-ML algorithm. The estimates and standard errors for the price duration data are more markedly different between the two methods than for volume durations. Moreover, the improved match between the data dispersion index and the implied one of the EIS-ML estimates is even more striking in this case. To draw a conclusion, provided that the model is correctly specified, by using EIS-ML estimation rather than QML, the estimates one gets are more precise.

Finally, in both table, in square brackets, we present the MC standard deviations of the EIS estimates. These standard deviations are calculated from ten different different estimates obtained by running the algorithm using each time a different random seed for the common random numbers employed in the EIS evaluation of the likelihood. The resulting dispersion of the estimates is extremely low, which suggests that EIS is a rather robust method in this respect. 


\section{Extensions}

In order to illustrate the flexibility of EIS-ML as a numerical tool, we estimate two extensions of the the SCD model described and used in the previous sections. The first extension consists in the introduction of a "leverage" term in the mean of the latent factor. For a motivation of this effect, we refer the reader to Feng, Jiang, and Song (2004), but we slightly differ from these authors by letting the value of $\psi_{i}$ to depend on the lagged duration $x_{i-1}$ (rather than $\left.\epsilon_{i-1}\right)$, such that equation (2) becomes

$$
\psi_{i}=\omega+\beta \psi_{i-1}+\alpha x_{i-1}+u_{i}
$$

The introduction of the lagged observed duration requires just a slight modification of the code and the effect on the speed of the algorithm is negligible: the EIS based computation of the likelihood takes almost exactly the same time while of course the introduction of an extra parameter slows down the maximization routine. Table 9 presents the results of the estimation with the data sets employed in the previous section. The introduction of the lagged duration as an explanatory variable can be tested by the likelihood ratio for the null hypothesis $\alpha=0$. The p-values are reported in the table in curled brackets before the value of the likelihood at its maximum.

For volume durations, the estimated leverage coefficients do not display a clear sign pattern. Moreover, the results of the LR tests are mixed: we clearly reject the null only for Exxon, with a positive leverage effect, while in three other cases the evidence is mixed (pvalue around 0.05) and the effect is negative. In the case of Boeing, the leverage effect is clearly not significant. For price durations the results are clearly in favor of a negative leverage effect, except for the puzzling case of the Exxon stock, where the estimates are somewhat unusual (low $\beta$ and high $\sigma$ ).

In the second variant of the model we change the distribution of variable $\epsilon_{i}$, representing the baseline duration. The Weibull distribution is replaced by a generalized gamma one with parameters $(\nu, \gamma, c)$. The third parameter is a location parameter and, like in the case of the Weibull, it is chosen so that the random variable has a unitary mean (so that $c$ is a function of 
$\nu$ and $\gamma$ and therefore does not appear in the parameters to estimate). The density function of the generalized gamma is as follows:

$$
f_{G G}(\epsilon)=\frac{\gamma}{c^{\nu \gamma} \Gamma(\nu)} \epsilon^{\nu \gamma-1} \exp \left[-\left(\frac{\epsilon}{c}\right)^{\gamma}\right]
$$

and it can be easily seen that the Weibull density is a particular case, arising when $\nu=1$. Further information about this distribution is available in Bauwens and Giot (2001), who provide a detailed description of its characteristics.

The modifications in the computer code that were required to use this extension were even simpler than in the case of the leverage effect. We did not observe any speed impact on the likelihood computation, while, of course, its maximization was a tad slower because of the introduction of an extra parameter. The estimation results are available in table 10 . Volume durations modeling does not appear to improve consistently with the introduction of this richer baseline density. The p-values of the LR tests for the null hypothesis $\nu=1$ are seldom low (except for Ibm) as the values taken by the parameter $\nu$ tend to be rather close to 1. Different results are obtained with price durations. A significant departure from the Weibull is observed (estimates of $\nu$ are between 4.7 to 7.2) and gains in likelihood are consistent, to the point that the p-values of the LR tests for $\nu=1$ are always smaller than 0.001. For the price durations of the Exxon stock the EIS-ML algorithm delivers a value for the likelihood but the maximization routine failed to achieve strong convergence, regardless of the vector of initial parameters chosen as a starting point.

\section{Conclusion}

This paper describes a new approach, the EIS-ML, for the numerical estimation of SCD models, showing its capability to deliver more precise estimates than the approximate QML method. The performance EIS-ML are tested both in a simulated and a real case, giving good results in terms of precision of estimation at a cost of an acceptable loss in rapidity of the computation. The evidence from the estimation of simulated series suggests an uncomfortably poor performance of the QML estimator when the latent factor variance is low, while EIS-ML 
appears to be much more robust. The algorithm is applied to the estimation of a set of volume and price durations showing a remarkably low MC variance. Moreover, we observed a significant gain in the capability of the SCD model in reproducing the first two empirical moments of the data when QML is replaced by EIS-ML estimation. Further interesting extensions are explored in the use of EIS-ML for the estimation of richer specifications, such as the presence of a leverage effect in the autoregressive component or a more flexible distribution for the baseline duration.

\section{Appendix}

Proof: [Theorem 1]

Given (24) and the definition of $\zeta\left(\psi_{i}, a_{i}\right)$ following (25), the function $k\left(\psi_{i}, a_{i}\right)$ is given by

$$
\begin{aligned}
k\left(\psi_{i}, a_{i}\right)= & q\left(\psi_{i} \mid \psi_{i-1}, a_{i}\right) \zeta\left(\psi_{i}, a_{i}\right) \\
= & \frac{1}{\sigma \sqrt{2 \pi}} \exp \left\{-\frac{1}{2}\left[\left(\frac{1}{\sigma^{2}}-2 a_{2, i}\right) \psi_{i}^{2}-2\left(\frac{\omega+\beta \psi_{i-1}}{\sigma^{2}}+a_{1, i}\right) \psi_{i}\right.\right. \\
& \left.\left.\quad+\left(\frac{\omega+\beta \psi_{i-1}}{\sigma}\right)^{2}\right]\right\} .
\end{aligned}
$$

Integrating $k\left(\psi_{i}, a_{i}\right)$ with respect to $\psi_{i}$, we obtain the function $\chi\left(\psi_{i-1}, a_{i}\right)$ given in (27). If we combine (30) and (27) as in (21), we find the functional form of $m\left(\psi_{i} \mid \psi_{i-1}, a_{i}\right)$, that can be easily rewritten as a Gaussian density with mean and variance equal to $\mu_{i}$ and $v_{i}^{2}$ as defined 
in $(26)$ :

$$
\begin{aligned}
m\left(\psi_{i} \mid \psi_{i-1}, a_{i}\right)= & k\left(\psi_{i}, a_{i}\right) / \chi\left(\psi_{i-1}, a_{i}\right) \\
= & \frac{\sqrt{1-2 \sigma^{2} a_{2, i}}}{\sigma \sqrt{2 \pi}} \exp \left\{-\frac{1}{2}\left[\left(\frac{1}{\sigma^{2}}-2 a_{2, i} \psi_{i}^{2}\right)\right.\right. \\
& -2\left(\frac{\omega+\beta \psi_{i-1}}{\sigma^{2}}-a_{1, i}\right) \psi_{i} \\
& \left.\left.+\frac{\sigma^{2}}{1-2 \sigma^{2} a_{2, i}}\left(\frac{\omega+\beta \psi_{i-1}}{\sigma_{2}}+a_{1, i}\right)^{2}\right]\right\} \\
= & \frac{1}{v_{i} \sqrt{2 \pi}} \exp \left\{-\frac{1}{2}\left[\frac{1}{v_{i}^{2}} \psi_{i}^{2}-2 \frac{\mu_{i}}{v_{i}^{2}} \psi_{i}+\frac{\mu_{i}^{2}}{v_{i}^{2}}\right]\right\} \\
= & \frac{1}{v_{i} \sqrt{2 \pi}} \exp \left[-\frac{1}{2 v_{i}^{2}}\left(\psi_{i}-\mu_{i}\right)^{2}\right] .
\end{aligned}
$$

\section{References}

Bauwens, L., And P. Giot (2001): Econometric modelling of stock market intraday activity. Kluwer Academic Publishers.

Bauwens, L., and N. Hautsch (2006): "Stochastic conditional intensity processes," Journal of Financial Econometrics, 4, 450-493.

Bauwens, L., And J. Rombouts (2004): "Econometrics," in Handbook of Computational Statistics, ed. by J. Gentle, W. Hrdle, and Y. Mori, vol. 1. Springer Verlag.

Bauwens, L., And D. Veredas (2004): "The stochastic conditional duration model: a latent factor model for the analysis of financial durations," Journal of Econometrics, 119(2), $381-412$.

C. Strickland, C. F., and G. Martin (2006): "Bayesian analysis of the stochastic conditional duration model," Computational Statistics and Data Analysis, 50(9), 2247-2267.

E. Ghysels, A. H., And E. Renault (1996): "Stochastic volatility," in Handbook of Statistics, ed. by G. Maddala, and C. Rao, vol. 14. Elsevier Sciences, Amsterdam. 
Engle, R., And J. R. Russell (1998): “Autoregessive conditional duration: a new approach foor irregularly spaced transaction data," Econometrica, 66, 1127-1162.

Feng, D., G. JiAng, And P. Song (2004): "Stochastic conditional duration models with leverage effect for financial transaction data," Journal of Financial Econometrics, 2, 390:421.

Liesenfeld, R., AND J. F. Richard (2003): "Univariate and multivariate stochastic volatility models: estimation and diagnostics," Journal of Empirical Finance, 10(4), 505-531.

NING, Q. (2004): "Estimation of the stochastic conditional duration model via alternative methods - ECF and GMM," mimeo.

Richard, J. F., AND W. Zhang (1998): "Efficient high-dimensional Monte Carlo importance sampling," Mimeo. Department of Economics. University of Pittsburgh. Forthcoming in the Journal of Econometrics. 
Table 1: Sampling means, standard deviations and mean-squared errors of 1000 estimates of the SCD model parameters for simulated series of 250 observations

\begin{tabular}{cc|cc|cc|cc}
\hline \hline 250 & & & & & \\
& DGP & Mean EIS & Mean QML & StDev EIS & StDev QML & MSE EIS & MSE QML \\
\hline & & & & & & & \\
$\omega$ & $\mathbf{0 . 0 0 0 0}$ & 0.0064 & 0.0000 & 0.0325 & 0.0649 & 0.0010 & 0.0042 \\
$\beta$ & $\mathbf{0 . 9 0 0 0}$ & 0.8262 & 0.7601 & 0.1482 & 0.2958 & 0.0274 & 0.1071 \\
$\sigma$ & $\mathbf{0 . 2 0 0 0}$ & 0.2431 & 0.2771 & 0.1073 & 0.1939 & 0.0133 & 0.0435 \\
$\gamma$ & $\mathbf{1 . 1 0 0 0}$ & 1.1261 & 1.1823 & 0.0792 & 0.4732 & 0.0069 & 0.2307 \\
$\omega$ & $\mathbf{0 . 0 0 0 0}$ & 0.0083 & -0.0332 & 0.0381 & 0.1373 & 0.0015 & 0.0199 \\
$\beta$ & $\mathbf{0 . 9 0 0 0}$ & 0.5963 & 0.0941 & 0.2784 & 0.5847 & 0.1697 & 0.9912 \\
$\sigma$ & $\mathbf{0 . 0 5 0 0}$ & 0.1703 & 0.3521 & 0.1384 & 0.3583 & 0.0336 & 0.2197 \\
$\gamma$ & $\mathbf{1 . 1 0 0 0}$ & 1.1415 & 1.5280 & 0.0801 & 1.2059 & 0.0081 & 1.6373 \\
$\omega$ & $\mathbf{0 . 0 0 0 0}$ & 0.01471 & -0.0729 & 0.0534 & 0.2164 & 0.0030 & 0.0521 \\
$\beta$ & $\mathbf{0 . 9 0 0 0}$ & 0.62392 & -0.0906 & 0.2828 & 0.5489 & 0.1562 & 1.2827 \\
$\sigma$ & $\mathbf{0 . 0 5 0 0}$ & 0.18714 & 0.5391 & 0.1982 & 0.5122 & 0.0581 & 0.5016 \\
$\gamma$ & $\mathbf{0 . 8 0 0 0}$ & 0.83921 & 1.3096 & 0.0779 & 1.4544 & 0.0076 & 2.3752 \\
$\omega$ & $\mathbf{0 . 0 0 0 0}$ & 0.0042 & -0.0235 & 0.0482 & 0.1411 & 0.0023 & 0.0204 \\
$\beta$ & $\mathbf{0 . 9 0 0 0}$ & 0.7804 & 0.4938 & 0.2092 & 0.5288 & 0.0580 & 0.4446 \\
$\sigma$ & $\mathbf{0 . 2 0 0 0}$ & 0.2734 & 0.4394 & 0.1657 & 0.3681 & 0.0328 & 0.1928 \\
$\gamma$ & $\mathbf{0 . 8 0 0 0}$ & 0.8261 & 0.9536 & 0.0634 & 0.6779 & 0.0047 & 0.4831 \\
\hline \hline
\end{tabular}

Table 2: Sampling means, standard deviations and mean-squared errors of 1000 estimates of the SCD model parameters for simulated series of 500 observations

\begin{tabular}{|c|c|c|c|c|c|c|c|}
\hline 500 & DGP & Mean EIS & Mean QML & StDev EIS & StDev QML & $M S E E I S$ & MSE QML \\
\hline$\omega$ & 0.0000 & 0.0038 & -0.0021 & 0.0146 & 0.0255 & 0.0002 & 0.0006 \\
\hline$\beta$ & 0.9000 & 0.8699 & 0.8467 & 0.0770 & 0.1476 & 0.0068 & 0.0246 \\
\hline$\sigma$ & 0.2000 & 0.2186 & 0.2357 & 0.0706 & 0.1149 & 0.0053 & 0.0144 \\
\hline$\gamma$ & 1.1000 & 1.1089 & 1.1202 & 0.0535 & 0.0778 & 0.0029 & 0.0064 \\
\hline$\omega$ & 0.0000 & 0.0043 & -0.0433 & 0.0255 & 0.1190 & 0.0006 & 0.0160 \\
\hline$\beta$ & 0.9000 & 0.6200 & 0.0981 & 0.2776 & 0.5902 & 0.1554 & 0.9913 \\
\hline$\sigma$ & 0.0500 & 0.1430 & 0.3270 & 0.1132 & 0.3392 & 0.0214 & 0.1918 \\
\hline$\gamma$ & 1.1000 & 1.1254 & 1.4719 & 0.0562 & 1.1315 & 0.0038 & 1.4187 \\
\hline$\omega$ & 0.0000 & 0.0068 & -0.0701 & 0.0368 & 0.1831 & 0.0014 & 0.0384 \\
\hline$\beta$ & 0.9000 & 0.6602 & -0.0944 & 0.2700 & 0.5288 & 0.1303 & 1.2686 \\
\hline$\sigma$ & 0.0500 & 0.1483 & 0.4989 & 0.1534 & 0.4563 & 0.0332 & 0.4098 \\
\hline$\gamma$ & 0.8000 & 0.8224 & 1.2012 & 0.0584 & 1.2614 & 0.0039 & 1.7521 \\
\hline$\omega$ & 0.0000 & 0.0035 & -0.0221 & 0.0261 & 0.0994 & 0.0006 & 0.0103 \\
\hline$\beta$ & 0.9000 & $0.844^{2}$ & 0.6473 & 0.1308 & 0.4308 & 0.0202 & 0.2494 \\
\hline$\sigma$ & 0.2000 & 0.2372 & 0.3708 & 0.1119 & 0.3157 & 0.0139 & 0.1289 \\
\hline$\gamma$ & 0.8000 & 0.8109 & 0.8863 & 0.0428 & 0.4887 & 0.0019 & 0.2462 \\
\hline
\end{tabular}


Table 3: Sampling means, standard deviations and mean-squared errors of 1000 estimates of the SCD model parameters for simulated series of 1000 observations

\begin{tabular}{cc|cc|cc|cc}
\hline \hline $\mathbf{1 0 0 0}$ & & & & & \\
& DGP & Mean EIS & Mean QML & StDev EIS & StDev QML & MSE EIS & MSE QML \\
\hline & & & & & & & \\
$\omega$ & $\mathbf{0 . 0 0 0 0}$ & 0.0019 & 0.0001 & 0.0090 & 0.0101 & 0.0000 & 0.0001 \\
$\beta$ & $\mathbf{0 . 9 0 0 0}$ & 0.8859 & 0.8803 & 0.0423 & 0.0626 & 0.0019 & 0.0043 \\
$\sigma$ & $\mathbf{0 . 2 0 0 0}$ & 0.2099 & 0.2147 & 0.0428 & 0.0674 & 0.0019 & 0.0047 \\
$\gamma$ & $\mathbf{1 . 1 0 0 0}$ & 1.1037 & 1.1098 & 0.0352 & 0.0414 & 0.0012 & 0.0018 \\
& & & & & & & \\
$\omega$ & $\mathbf{0 . 0 0 0 0}$ & 0.0012 & -0.0516 & 0.0166 & 0.1122 & 0.0002 & 0.0152 \\
$\beta$ & $\mathbf{0 . 9 0 0 0}$ & 0.6883 & 0.1722 & 0.2571 & 0.5722 & 0.1109 & 0.8570 \\
$\sigma$ & $\mathbf{0 . 0 5 0 0}$ & 0.1074 & 0.3315 & 0.0882 & 0.3381 & 0.0110 & 0.1935 \\
$\gamma$ & $\mathbf{1 . 1 0 0 0}$ & 1.1133 & 1.4830 & 0.0365 & 1.1450 & 0.0015 & 1.4579 \\
$\omega$ & $\mathbf{0 . 0 0 0 0}$ & 0.0018 & -0.0915 & 0.0198 & 0.1822 & 0.0003 & 0.0415 \\
$\beta$ & $\mathbf{0 . 9 0 0 0}$ & 0.7376 & -0.0241 & 0.2367 & 0.5091 & 0.0823 & 1.1131 \\
$\sigma$ & $\mathbf{0 . 0 5 0 0}$ & 0.1056 & 0.5402 & 0.1166 & 0.4680 & 0.0167 & 0.4594 \\
$\gamma$ & $\mathbf{0 . 8 0 0 0}$ & 0.8111 & 1.2733 & 0.0383 & 1.3596 & 0.0015 & 2.0726 \\
$\omega$ & $\mathbf{0 . 0 0 0 0}$ & 0.0016 & -0.009 & 0.0187 & 0.0514 & 0.0003 & 0.0027 \\
$\beta$ & $\mathbf{0 . 9 0 0 0}$ & 0.8712 & 0.7818 & 0.0957 & 0.2838 & 0.0100 & 0.0945 \\
$\sigma$ & $\mathbf{0 . 2 0 0 0}$ & 0.2183 & 0.2974 & 0.0718 & 0.2360 & 0.0055 & 0.0652 \\
$\gamma$ & $\mathbf{0 . 8 0 0 0}$ & 0.8043 & 0.8285 & 0.0300 & 0.1498 & 0.0009 & 0.0232 \\
\hline \hline
\end{tabular}

Table 4: Sampling means, standard deviations and mean-squared errors of 1000 estimates of the SCD model parameters for simulated series of 5000 observations

\begin{tabular}{|c|c|c|c|c|c|c|c|}
\hline 5000 & DGP & Mean EIS & Mean QML & StDev EIS & StDev QML & $M S E E I S$ & MSE QML \\
\hline$\omega$ & 0.0000 & 0.0005 & 0.0002 & 0.0031 & 0.0034 & 0.0000 & 0.0000 \\
\hline$\beta$ & 0.9000 & 0.8982 & 0.8959 & 0.0152 & 0.0201 & 0.0002 & 0.0004 \\
\hline$\sigma$ & 0.2000 & 0.1999 & 0.2035 & 0.0175 & 0.0263 & 0.0003 & 0.0007 \\
\hline$\gamma$ & 1.1000 & 1.0994 & 1.1026 & 0.0146 & 0.0173 & 0.0002 & 0.0003 \\
\hline$\omega$ & 0.0000 & -0.0002 & -0.0302 & 0.0052 & 0.0703 & 0.0000 & 0.0058 \\
\hline$\beta$ & 0.9000 & 0.7793 & 0.3099 & 0.2142 & 0.4937 & 0.0604 & 0.5919 \\
\hline$\sigma$ & 0.0500 & 0.0785 & 0.2934 & 0.0522 & 0.2516 & 0.0035 & 0.1225 \\
\hline$\gamma$ & 1.1000 & 1.1048 & 1.2761 & 0.0162 & 0.7875 & 0.0002 & 0.6512 \\
\hline$\omega$ & 0.0000 & -0.0003 & -0.0767 & 0.0068 & 0.1265 & 0.0000 & 0.0219 \\
\hline$\beta$ & 0.9000 & 0.7893 & 0.0781 & 0.2135 & 0.3672 & 0.0578 & 0.8103 \\
\hline$\sigma$ & 0.0500 & 0.0786 & 0.5901 & 0.0755 & 0.3513 & 0.0065 & 0.4152 \\
\hline$\gamma$ & 0.8000 & 0.8033 & 1.0754 & 0.0154 & 1.0024 & 0.0002 & 1.0807 \\
\hline$\omega$ & 0.0000 & 0.0003 & -0.0001 & 0.0047 & 0.0069 & 0.0000 & 0.0000 \\
\hline$\beta$ & 0.9000 & 0.8931 & 0.8909 & 0.0380 & 0.0536 & 0.0014 & 0.0029 \\
\hline$\sigma$ & 0.2000 & 0.2024 & 0.2086 & 0.0295 & 0.0612 & 0.0008 & 0.0038 \\
\hline$\gamma$ & 0.8000 & 0.8001 & 0.8023 & 0.0109 & 0.0158 & 0.0001 & 0.0002 \\
\hline
\end{tabular}


Table 5: Sampling means, standard deviations and mean-squared errors of 1000 estimates of the SCD model parameters for simulated series of 10000 observations

\begin{tabular}{|c|c|c|c|c|c|c|c|}
\hline 10000 & DGP & Mean EIS & Mean QML & StDev EIS & StDev QML & $M S E$ EIS & MSE QML \\
\hline$\omega$ & 0.0000 & $<0.0001$ & 0.0001 & 0.0021 & 0.0024 & $<0.0000$ & $<0.0000$ \\
\hline$\beta$ & 0.9000 & 0.9005 & 0.8999 & 0.0119 & 0.0139 & 0.0001 & 0.0002 \\
\hline$\sigma$ & 0.2000 & 0.1981 & 0.1986 & 0.0134 & 0.0192 & 0.0002 & 0.0004 \\
\hline$\gamma$ & 1.1000 & 1.0999 & 1.1002 & 0.0098 & 0.0114 & $<0.0000$ & 0.0001 \\
\hline$\omega$ & 0.0000 & -0.0002 & -0.0307 & 0.0021 & 0.0278 & $<0.0000$ & 0.0044 \\
\hline$\beta$ & 0.9000 & 0.8561 & 0.2351 & 0.1145 & 0.3954 & 0.0150 & 0.5985 \\
\hline$\sigma$ & 0.0500 & 0.0615 & 0.3606 & 0.0299 & 0.1944 & 0.0010 & 0.1342 \\
\hline$\gamma$ & 1.1000 & 1.1018 & 1.1761 & 0.0092 & 0.0701 & $<0.0000$ & 0.0107 \\
\hline$\omega$ & 0.0000 & -0.0001 & -0.0867 & 0.0024 & 0.1213 & $<0.0000$ & 0.0222 \\
\hline$\beta$ & 0.9000 & 0.8411 & 0.0522 & 0.1481 & 0.3294 & 0.0254 & 0.8273 \\
\hline$\sigma$ & 0.0500 & 0.0624 & 0.6381 & 0.0432 & 0.3477 & 0.0020 & 0.4668 \\
\hline$\gamma$ & 0.8000 & 0.8013 & 1.0656 & 0.0068 & 0.8636 & $<0.0000$ & 0.8164 \\
\hline$\omega$ & 0.0000 & -0.0003 & $<0.0001$ & 0.0025 & 0.0027 & $<0.0000$ & $<0.0000$ \\
\hline$\beta$ & 0.9000 & 0.8981 & 0.9001 & 0.0154 & 0.0221 & 0.0002 & 0.0005 \\
\hline$\sigma$ & 0.2000 & 0.2010 & 0.1992 & 0.0190 & 0.0307 & 0.0004 & 0.0009 \\
\hline$\gamma$ & 0.8000 & 0.8005 & 0.7994 & 0.0069 & 0.0081 & $<0.0000$ & $<0.0000$ \\
\hline
\end{tabular}

Table 6: Sampling means, standard deviations and mean-squared errors of 1000 estimates of the SCD model parameters for simulated series of one million observations

\begin{tabular}{cc|c|c|c|c}
\hline \hline \multirow{2}{*}{$\mathbf{1 0 0 0 0 0 0}$} & & & & \\
& DGP & Mean QML & StDev QML & Min QML & Max QML \\
\hline$\omega$ & $\mathbf{0 . 0 0 0 0}$ & -0.0001 & 0.0002 & -0.0004 & 0.0003 \\
$\beta$ & $\mathbf{0 . 9 0 0 0}$ & 0.9001 & 0.0007 & 0.8989 & 0.9012 \\
$\sigma$ & $\mathbf{0 . 2 0 0 0}$ & 0.1996 & 0.0013 & 0.1979 & 0.2013 \\
$\gamma$ & $\mathbf{1 . 1 0 0 0}$ & 1.1009 & 0.0008 & 1.0992 & 1.1022 \\
$\omega$ & & & & & \\
$\beta$ & $\mathbf{0 . 0 0 0 0}$ & -0.0001 & 0.0001 & -0.0002 & 0.0002 \\
$\sigma$ & $\mathbf{0 . 9 0 0 0}$ & 0.8997 & 0.0078 & 0.8875 & 0.9125 \\
$\gamma$ & $\mathbf{0 . 0 5 0 0}$ & 0.0496 & 0.0031 & 0.0445 & 0.0549 \\
& $\mathbf{1 . 1 0 0 0}$ & 1.1008 & 0.0011 & 1.0985 & 1.1025 \\
$\omega$ & & & & & \\
$\beta$ & $\mathbf{0 . 0 0 0 0}$ & 0.0000 & 0.0001 & -0.0003 & 0.0002 \\
$\sigma$ & $\mathbf{0 . 9 0 0 0}$ & 0.9006 & 0.0129 & 0.8787 & 0.9201 \\
$\gamma$ & $\mathbf{0 . 0 5 0 0}$ & 0.04911 & 0.0053 & 0.0413 & 0.0581 \\
& $\mathbf{0 . 8 0 0 0}$ & 0.8007 & 0.0007 & 0.7993 & 0.8018 \\
$\omega$ & & & & & \\
$\beta$ & $\mathbf{0 . 0 0 0 0}$ & 0.0000 & 0.0003 & -0.0004 & 0.0004 \\
$\sigma$ & $\mathbf{0 . 9 0 0 0}$ & 0.9002 & 0.0012 & 0.8985 & 0.9018 \\
$\gamma$ & $\mathbf{0 . 2 0 0 0}$ & 0.1993 & 0.0020 & 0.1966 & 0.2027 \\
& $\mathbf{0 . 8 0 0 0}$ & 0.8007 & 0.0006 & 0.7994 & 0.8017 \\
\hline \hline
\end{tabular}


Table 7: Results for volume durations for an SCD model

\begin{tabular}{|c|c|c|c|c|c|c|}
\hline VOLUME & & Boeing & Coca Cola & Disney & Exxon & $\mathrm{Ibm}$ \\
\hline obs. & & 1576 & 3022 & 1778 & 2045 & 4305 \\
\hline \multirow[t]{2}{*}{$\omega$} & $q m l$ & $\begin{array}{c}0.002 \\
(0.0037)\end{array}$ & $\begin{array}{c}-0.001 \\
(0.0053)\end{array}$ & $\begin{array}{c}0.000 \\
(0.0029)\end{array}$ & $\begin{array}{c}0.007 \\
(0.0044)\end{array}$ & $\begin{array}{c}0.000 \\
(0.0016)\end{array}$ \\
\hline & eis & $\begin{array}{c}0.002 \\
(0.0026) \\
{[<0.0001]}\end{array}$ & $\begin{array}{c}0.002 \\
(0.0021) \\
{[<0.0001]}\end{array}$ & $\begin{array}{c}0.002 \\
(0.0018) \\
{[0.0001]}\end{array}$ & $\begin{array}{c}0.006 \\
(0.0030) \\
{[<0.0001]}\end{array}$ & $\begin{array}{l}-0.002 \\
(0.0010) \\
{[0.0001]}\end{array}$ \\
\hline \multirow[t]{2}{*}{$\beta$} & $q m l$ & $\begin{array}{c}0.921 \\
(0.0129)\end{array}$ & $\begin{array}{c}0.865 \\
(0.0276)\end{array}$ & $\begin{array}{c}0.976 \\
(0.0074)\end{array}$ & $\begin{array}{c}0.901 \\
(0.0237)\end{array}$ & $\begin{array}{c}0.982 \\
(0.0044)\end{array}$ \\
\hline & eis & $\begin{array}{c}0.961 \\
(0.0116) \\
{[<0.0001]}\end{array}$ & $\begin{array}{c}0.950 \\
(0.0129) \\
{[<0.0001]}\end{array}$ & $\begin{array}{c}0.981 \\
(0.0061) \\
{[<0.0001]}\end{array}$ & $\begin{array}{c}0.925 \\
(0.0218) \\
{[<0.0001]}\end{array}$ & $\begin{array}{c}0.982 \\
(0.0032) \\
{[0.0005]}\end{array}$ \\
\hline \multirow[t]{2}{*}{$\sigma$} & $q m l$ & $\begin{array}{c}0.116 \\
(0.0168)\end{array}$ & $\begin{array}{c}0.209 \\
(0.0278)\end{array}$ & $\begin{array}{c}0.102 \\
(0.0117)\end{array}$ & $\begin{array}{c}0.133 \\
(0.0206)\end{array}$ & $\begin{array}{c}0.101 \\
(0.0020)\end{array}$ \\
\hline & eis & $\begin{array}{c}0.101 \\
(0.0143) \\
{[<0.0001]}\end{array}$ & $\begin{array}{c}0.109 \\
(0.0159) \\
{[<0.0001]}\end{array}$ & $\begin{array}{c}0.088 \\
(0.0101) \\
{[<0.0001]}\end{array}$ & $\begin{array}{c}0.102 \\
(0.0181) \\
{[<0.0001]}\end{array}$ & $\begin{array}{c}0.112 \\
(0.0081) \\
{[0.0002]}\end{array}$ \\
\hline \multirow[t]{2}{*}{$\gamma$} & $q m l$ & $\begin{array}{c}1.698 \\
(0.0288)\end{array}$ & $\begin{array}{c}1.401 \\
(0.0219)\end{array}$ & $\begin{array}{c}1.767 \\
(0.0259)\end{array}$ & $\begin{array}{c}1.812 \\
(0.0310)\end{array}$ & $\begin{array}{c}1.892 \\
(0.0238)\end{array}$ \\
\hline & eis & $\begin{array}{l}1.713 \\
(0.0384) \\
{[0.0001]}\end{array}$ & $\begin{array}{c}1.310 \\
(0.0215) \\
{[<0.0001]}\end{array}$ & $\begin{array}{c}1.777 \\
(0.0368) \\
{[0.0006]}\end{array}$ & $\begin{array}{c}1.764 \\
(0.0375) \\
{[<0.0001]}\end{array}$ & $\begin{array}{c}1.837 \\
(0.0266) \\
{[0.0005]}\end{array}$ \\
\hline eis log lik & & $\begin{array}{r}-1303.51 \\
{[0.0186]}\end{array}$ & $\begin{array}{r}-2843.79 \\
{[0.0062]}\end{array}$ & $\begin{array}{c}-1381.95 \\
{[0.0450]}\end{array}$ & $\begin{array}{r}-1682.94 \\
{[0.0116]}\end{array}$ & $\begin{array}{r}-3158.13 \\
{[0.5885]}\end{array}$ \\
\hline \multirow[t]{3}{*}{$\tilde{\delta}_{x}$} & data & 0.70 & 0.88 & 0.72 & 0.65 & 0.80 \\
\hline & $\mathrm{qml}$ & 0.76 & 0.90 & 0.82 & 0.67 & 0.86 \\
\hline & eis & 0.74 & 0.89 & 0.80 & 0.66 & 0.94 \\
\hline
\end{tabular}

QML and EIS-ML estimates and standard errors in parentheses. MC standard deviations for the EIS estimates are in square parentheses. $\tilde{\delta}_{x}$ denotes the dispersion index. The estimated model is defined by (1)-(4) and (23). 
Table 8: Results for price durations for an SCD model

\begin{tabular}{|c|c|c|c|c|c|c|}
\hline PRICE & & Boeing & Coca ola & Disney & Exxon & Ibm \\
\hline obs. & & 2620 & 1609 & 2160 & 2717 & 6728 \\
\hline \multirow[t]{2}{*}{$\omega$} & $q m l$ & $\begin{array}{c}-0.026 \\
(0.0081)\end{array}$ & $\begin{array}{c}-0.035 \\
(0.0166)\end{array}$ & $\begin{array}{c}-0.005 \\
(0.0030)\end{array}$ & $\begin{array}{c}0.008 \\
(0.0047)\end{array}$ & $\begin{array}{c}-0.005 \\
(0.0020)\end{array}$ \\
\hline & eis & $\begin{array}{l}-0.023 \\
(0.0097) \\
{[0.0001]}\end{array}$ & $\begin{array}{c}-0.027 \\
(0.0154) \\
{[<0.0001]}\end{array}$ & $\begin{array}{c}-0.002 \\
(0.0033) \\
{[<0.0001]}\end{array}$ & $\begin{array}{c}-0.127 \\
(0.0243) \\
{[<0.0001]}\end{array}$ & $\begin{array}{c}-0.006 \\
(0.0028) \\
{[<0.0001]}\end{array}$ \\
\hline \multirow[t]{2}{*}{$\beta$} & $q m l$ & $\begin{array}{c}0.896 \\
(0.0194)\end{array}$ & $\begin{array}{c}(0.774) \\
(0.0770)\end{array}$ & $\begin{array}{c}0.967 \\
(0.0103)\end{array}$ & $\begin{array}{c}0.921 \\
(0.0356)\end{array}$ & $\begin{array}{c}0.977 \\
(0.0051)\end{array}$ \\
\hline & eis & $\begin{array}{c}0.876 \\
(0.0302) \\
{[0.0004]}\end{array}$ & $\begin{array}{c}0.733 \\
(0.0731) \\
{[0.0006]}\end{array}$ & $\begin{array}{c}0.960 \\
(0.0134) \\
{[<0.0001]}\end{array}$ & $\begin{array}{c}0.179 \\
(0.0564) \\
{[0.0005]}\end{array}$ & $\begin{array}{c}0.962 \\
(0.0074) \\
{[<0.0001]}\end{array}$ \\
\hline \multirow[t]{2}{*}{$\sigma$} & $q m l$ & $\begin{array}{c}0.286 \\
(0.0301)\end{array}$ & $\begin{array}{c}0.292 \\
(0.0739)\end{array}$ & $\begin{array}{c}0.108 \\
(0.0181)\end{array}$ & $\begin{array}{c}0.100 \\
(0.0320)\end{array}$ & $\begin{array}{c}0.135 \\
(0.0041)\end{array}$ \\
\hline & eis & $\begin{array}{c}0.332 \\
(0.0487) \\
{[0.0008]}\end{array}$ & $\begin{array}{c}0.377 \\
(0.0698) \\
{[0.0006]}\end{array}$ & $\begin{array}{c}0.136 \\
(0.0247) \\
{[<0.0001]}\end{array}$ & $\begin{array}{c}0.674 \\
(0.0344) \\
{[0.0002]}\end{array}$ & $\begin{array}{c}0.192 \\
(0.0197) \\
{[0.0002]}\end{array}$ \\
\hline \multirow[t]{2}{*}{$\gamma$} & $q m l$ & $\begin{array}{c}1.149 \\
(0.0200)\end{array}$ & $\begin{array}{c}1.113 \\
(0.0308)\end{array}$ & $\begin{array}{c}1.177 \\
(0.0192)\end{array}$ & $\begin{array}{c}1.161 \\
(0.0175)\end{array}$ & $\begin{array}{c}1.244 \\
(0.0131)\end{array}$ \\
\hline & eis & $\begin{array}{c}1.067 \\
(0.0284) \\
{[0.0004]}\end{array}$ & $\begin{array}{l}1.113 \\
(0.0402) \\
{[0.0003]}\end{array}$ & $\begin{array}{c}1.056 \\
(0.0208) \\
{[<0.0001]}\end{array}$ & $\begin{array}{l}1.344 \\
(0.0493) \\
{[0.0003]}\end{array}$ & $\begin{array}{c}1.130 \\
(0.0159) \\
{[<0.0001]}\end{array}$ \\
\hline eis log lik & & $\begin{array}{c}-2371.96 \\
{[0.0336]}\end{array}$ & $\begin{array}{c}-1561.44 \\
{[0.0192]}\end{array}$ & $\begin{array}{r}-2056.21 \\
{[0.0041]}\end{array}$ & $\begin{array}{r}-2635.10 \\
{[0.0663]}\end{array}$ & $\begin{array}{r}-5760.00 \\
{[0.0404]}\end{array}$ \\
\hline \multirow[t]{3}{*}{$\tilde{\delta}_{x}$} & data & 1.36 & 1.21 & 1.23 & 1.23 & 1.43 \\
\hline & $\mathrm{qml}$ & 1.29 & 1.07 & 1.03 & 0.93 & 1.21 \\
\hline & eis & 1.42 & 1.21 & 1.19 & 1.23 & 1.40 \\
\hline
\end{tabular}

QML and EIS-ML estimates and standard errors in parentheses. MC standard deviations for the EIS estimates are in square parentheses. $\tilde{\delta}_{x}$ denotes the dispersion index. The estimated model is defined by (1)- 4 and 23 . 
Table 9: Results for volume and price durations for an SCD model with leverage

\begin{tabular}{|c|c|c|c|c|c|}
\hline VOLUME & Boeing & Coca Cola & Disney & Exxon & $\mathrm{Ibm}$ \\
\hline obs. & 1576 & 3022 & 1778 & 2045 & 4305 \\
\hline$\omega$ & $\begin{array}{l}-0.008 \\
(0.009)\end{array}$ & $\begin{array}{l}0.028 \\
(0.016)\end{array}$ & $\begin{array}{c}0.012 \\
(0.007)\end{array}$ & $\begin{array}{l}-0.065 \\
(0.021)\end{array}$ & $\begin{array}{l}0.004 \\
(0.002)\end{array}$ \\
\hline$\beta$ & $\begin{array}{l}0.954 \\
(0.016)\end{array}$ & $\begin{array}{c}0.972 \\
(0.014)\end{array}$ & $\begin{array}{c}0.991 \\
(0.005)\end{array}$ & $\begin{array}{l}0.867 \\
(0.031)\end{array}$ & $\begin{array}{l}0.988 \\
(0.002)\end{array}$ \\
\hline$\sigma$ & $\begin{array}{l}0.096 \\
(0.014)\end{array}$ & $\begin{array}{l}0.117 \\
(0.018)\end{array}$ & $\begin{array}{c}0.088 \\
(0.010)\end{array}$ & $\begin{array}{l}0.043 \\
(0.030)\end{array}$ & $\begin{array}{c}0.115 \\
(0.010)\end{array}$ \\
\hline$\gamma$ & $\begin{array}{l}1.700 \\
(0.039)\end{array}$ & $\begin{array}{l}1.330 \\
(0.025)\end{array}$ & $\begin{array}{c}1.78 \\
(0.037)\end{array}$ & $\begin{array}{l}1.674 \\
(0.040)\end{array}$ & $\begin{array}{l}1.847 \\
(0.028)\end{array}$ \\
\hline$\alpha$ & $\begin{array}{l}0.011 \\
(0.010)\end{array}$ & $\begin{array}{l}-0.028 \\
(0.016)\end{array}$ & $\begin{array}{c}-0.013 \\
(0.007)\end{array}$ & $\begin{array}{l}0.079 \\
(0.024)\end{array}$ & $\begin{array}{l}-0.007 \\
(0.002)\end{array}$ \\
\hline LR p-value & 0.289 & 0.068 & 0.041 & $<0.001$ & 0.049 \\
\hline EIS log-lik & -1302.96 & -2842.12 & -1379.86 & -1676.29 & -3156.17 \\
\hline PRICE & Boeing & Coca Cola & Disney & Exxon & $\mathrm{Ibm}$ \\
\hline obs. & 2620 & 1609 & 2160 & 2717 & 6728 \\
\hline$\omega$ & $\begin{array}{l}0.049 \\
(0.011)\end{array}$ & $\begin{array}{l}0.119 \\
(0.034)\end{array}$ & $\begin{array}{l}0.041 \\
(0.007)\end{array}$ & $\begin{array}{l}-0.120 \\
(0.077)\end{array}$ & $\begin{array}{l}0.009 \\
(0.002)\end{array}$ \\
\hline$\beta$ & $\begin{array}{l}0.937 \\
(0.023)\end{array}$ & $\begin{array}{l}0.883 \\
(0.055)\end{array}$ & $\begin{array}{l}0.986 \\
(0.005)\end{array}$ & $\begin{array}{l}0.193 \\
(0.153)\end{array}$ & $\begin{array}{c}0.971 \\
(0.006)\end{array}$ \\
\hline$\sigma$ & $\begin{array}{l}0.331 \\
(0.043)\end{array}$ & $\begin{array}{l}0.392 \\
(0.056)\end{array}$ & $\begin{array}{l}0.166 \\
(0.038)\end{array}$ & $\begin{array}{l}0.673 \\
(0.038)\end{array}$ & $\begin{array}{l}0.205 \\
(0.018)\end{array}$ \\
\hline$\gamma$ & $\begin{array}{l}1.098 \\
(0.027)\end{array}$ & $\begin{array}{l}1.192 \\
(0.042)\end{array}$ & $\begin{array}{l}1.095 \\
(0.022)\end{array}$ & $\begin{array}{l}1.344 \\
(0.050)\end{array}$ & $\begin{array}{l}1.143 \\
(0.015)\end{array}$ \\
\hline$\alpha$ & $\begin{array}{c}-0.063 \\
(0.011)\end{array}$ & $\begin{array}{l}-0.133 \\
(0.029)\end{array}$ & $\begin{array}{c}-0.048 \\
(0.009)\end{array}$ & $\begin{array}{l}-0.005 \\
(0.049)\end{array}$ & $\begin{array}{l}-0.016 \\
(0.003)\end{array}$ \\
\hline LR p-value & $<0.001$ & $<0.001$ & $<0.001$ & 0.924 & $<0.001$ \\
\hline EIS log-lik & -2361.79 & -1552.83 & -2041.77 & -2635.10 & -5748.14 \\
\hline
\end{tabular}

EIS-ML estimates of the parameters and standard errors in parentheses. The LR p-value is for the hypothesis $\alpha=0$. The estimated model is defined by $(1),(28), \sqrt{3}, \sqrt{4}$ and $(23)$. 
Table 10: Results for volume and price durations for an SCD model with a generalized gamma baseline duration

\begin{tabular}{|c|c|c|c|c|c|}
\hline VOLUME & Boeing & Coca Cola & Disney & Exxon & Ibm \\
\hline obs. & 1576 & 3022 & 1778 & 2045 & 4305 \\
\hline$\omega$ & $\begin{array}{l}0.006 \\
(0.005)\end{array}$ & $\begin{array}{l}-0.011 \\
(0.008)\end{array}$ & $\begin{array}{c}0.002 \\
(0.002)\end{array}$ & $\begin{array}{l}-0.001 \\
(0.008)\end{array}$ & $\begin{array}{l}-0.006 \\
(0.002)\end{array}$ \\
\hline$\beta$ & $\begin{array}{l}0.960 \\
(0.012)\end{array}$ & $\begin{array}{l}0.953 \\
(0.012)\end{array}$ & $\begin{array}{c}0.981 \\
(0.006)\end{array}$ & $\begin{array}{l}0.930 \\
(0.021)\end{array}$ & $\begin{array}{c}0.982 \\
(0.004)\end{array}$ \\
\hline$\sigma$ & $\begin{array}{l}0.104 \\
(0.016)\end{array}$ & $\begin{array}{c}0.104 \\
(0.016)\end{array}$ & $\begin{array}{c}0.089 \\
(0.010)\end{array}$ & $\begin{array}{l}0.098 \\
(0.018)\end{array}$ & $\begin{array}{l}0.104 \\
(0.008)\end{array}$ \\
\hline$\gamma$ & $\begin{array}{l}1.900 \\
(0.198)\end{array}$ & $\begin{array}{l}1.126 \\
(0.092)\end{array}$ & $\begin{array}{c}1.819 \\
(0.163)\end{array}$ & $\begin{array}{l}1.633 \\
(0.155)\end{array}$ & $\begin{array}{l}1.437 \\
(0.088)\end{array}$ \\
\hline$\nu$ & $\begin{array}{l}0.854 \\
(0.130)\end{array}$ & $\begin{array}{l}1.274 \\
(0.166)\end{array}$ & $\begin{array}{c}0.964 \\
(0.133)\end{array}$ & $\begin{array}{l}1.128 \\
(0.165)\end{array}$ & $\begin{array}{l}1.489 \\
(0.149)\end{array}$ \\
\hline LR p-value & 0.307 & 0.054 & 0.792 & 0.405 & $<0.001$ \\
\hline EIS log-lik & -1302.99 & -2841.94 & -1381.92 & -1682.59 & -3149.46 \\
\hline$\delta_{x}$ data & 0.70 & 0.88 & 0.72 & 0.65 & 0.80 \\
\hline$\delta_{x}$ eis & 0.74 & 0.91 & 0.81 & 0.67 & 0.90 \\
\hline PRICE & Boeing & Coca Cola & Disney & Exxon & Ibm \\
\hline obs. & 2620 & 1609 & 2160 & 2717 & 6728 \\
\hline$\omega$ & $\begin{array}{c}-0.377 \\
(0.151)\end{array}$ & $\begin{array}{l}-0.666 \\
(0.292)\end{array}$ & $\begin{array}{l}-0.170 \\
(0.077)\end{array}$ & na & $\begin{array}{c}-0.105 \\
(0.029)\end{array}$ \\
\hline$\beta$ & $\begin{array}{l}0.923 \\
(0.020)\end{array}$ & $\begin{array}{c}0.828 \\
(0.044)\end{array}$ & $\begin{array}{l}0.970 \\
(0.010)\end{array}$ & na & $\begin{array}{c}0.982 \\
(0.004)\end{array}$ \\
\hline$\sigma$ & $\begin{array}{l}0.235 \\
(0.367)\end{array}$ & $\begin{array}{l}0.250 \\
(0.046)\end{array}$ & $\begin{array}{l}0.106 \\
(0.019)\end{array}$ & na & $\begin{array}{c}0.123 \\
(0.013)\end{array}$ \\
\hline$\gamma$ & $\begin{array}{l}0.389 \\
(0.061)\end{array}$ & $\begin{array}{l}0.440 \\
(0.080)\end{array}$ & $\begin{array}{l}0.0365 \\
(0.057)\end{array}$ & na & $\begin{array}{l}0.373 \\
(0.031)\end{array}$ \\
\hline$\nu$ & $\begin{array}{c}5.631 \\
(1.665)\end{array}$ & $\begin{array}{l}4.717 \\
(1.583)\end{array}$ & $\begin{array}{c}6.894 \\
(2.058)\end{array}$ & na & $\begin{array}{l}7.167 \\
(1.151)\end{array}$ \\
\hline LR p-value & $<0.001$ & $<0.001$ & $<0.001$ & na & $<0.001$ \\
\hline EIS log-lik & -2335.20 & -1542.15 & -2009.12 & na & -5617.91 \\
\hline$\delta_{x}$ data & 1.36 & 1.21 & 1.23 & 1.23 & 1.43 \\
\hline$\delta_{x}$ eis & 1.57 & 1.32 & 1.33 & na & 1.52 \\
\hline
\end{tabular}

EIS-ML estimates of the parameters and standard errors in parentheses. The LR p-value is for the hypothesis $\nu=1$. The estimated model is defined by (1)-(4) and (29). For Exxon price durations, results are not available (na). 

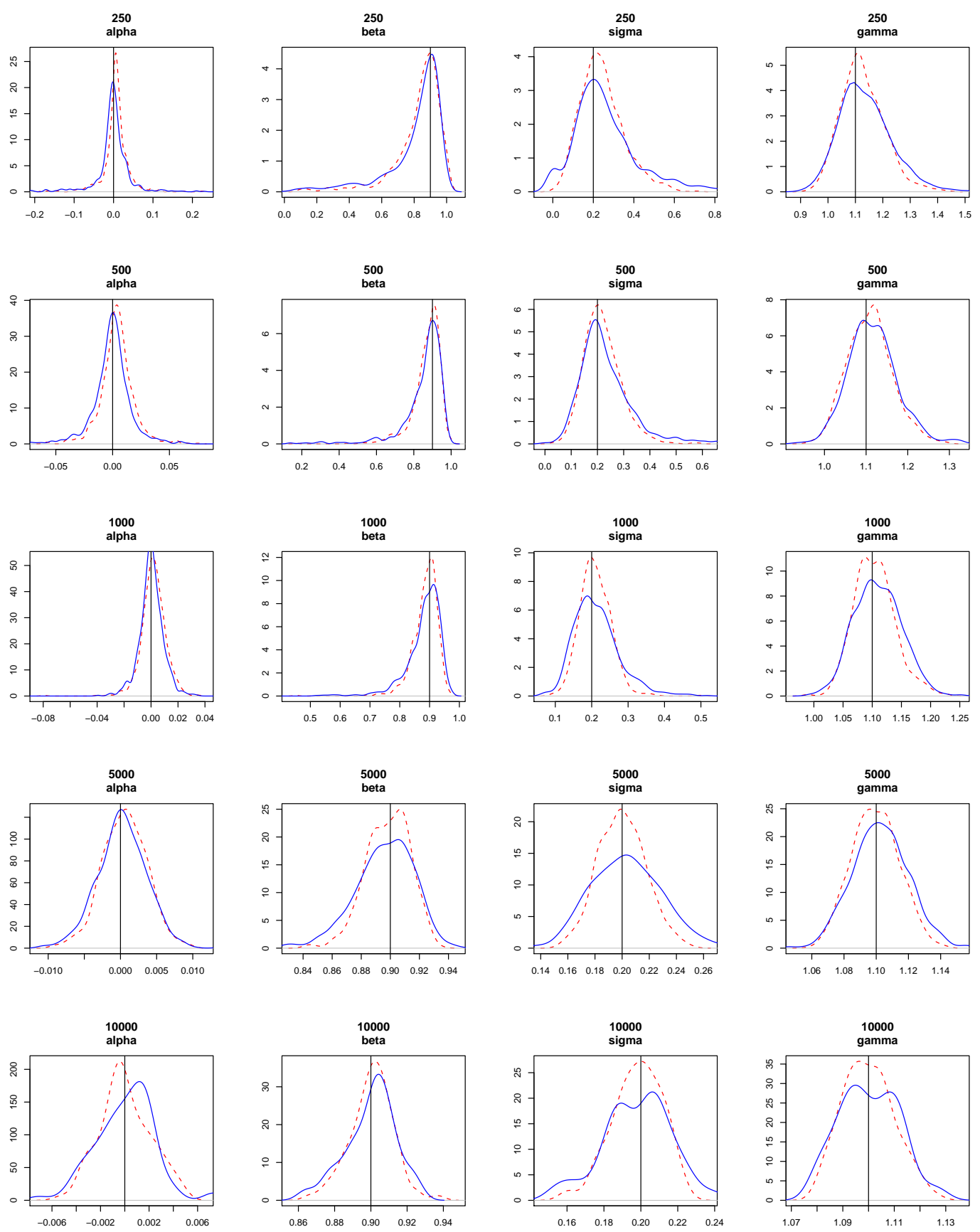

Figure 1: Sampling densities of 1000 EIS-ML (red, dashed) and QML (blue, full) estimates of the parameters of an SCD model with parameters $\omega=0.0, \beta=0.9, \sigma=0.2, \gamma=1.1$. 

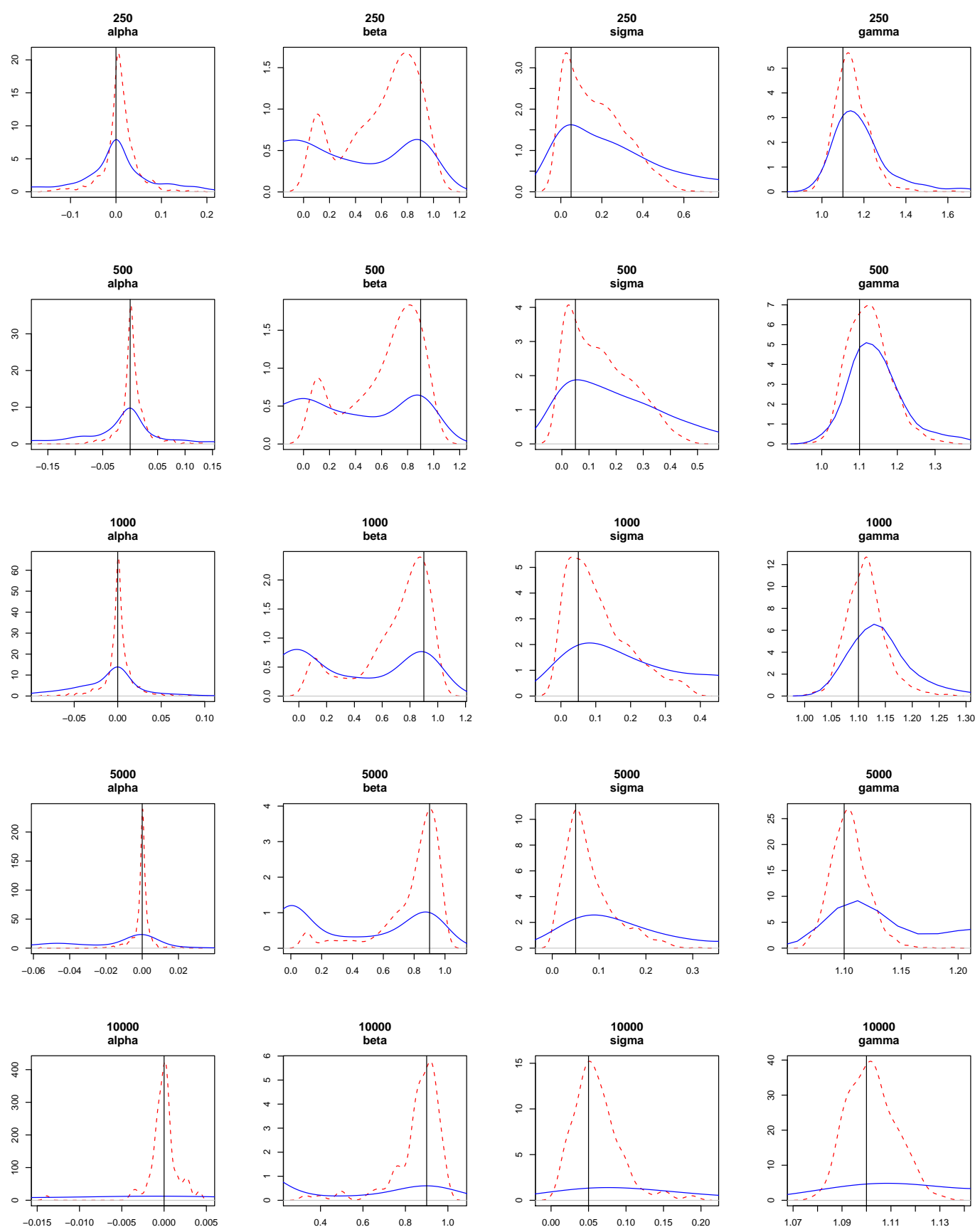

Figure 2: Sampling densities of 1000 EIS-ML (red, dashed) and QML (blue, full) estimates of the parameters of an SCD model with parameters $\omega=0.0, \beta=0.9, \sigma=0.05, \gamma=1.1$. 

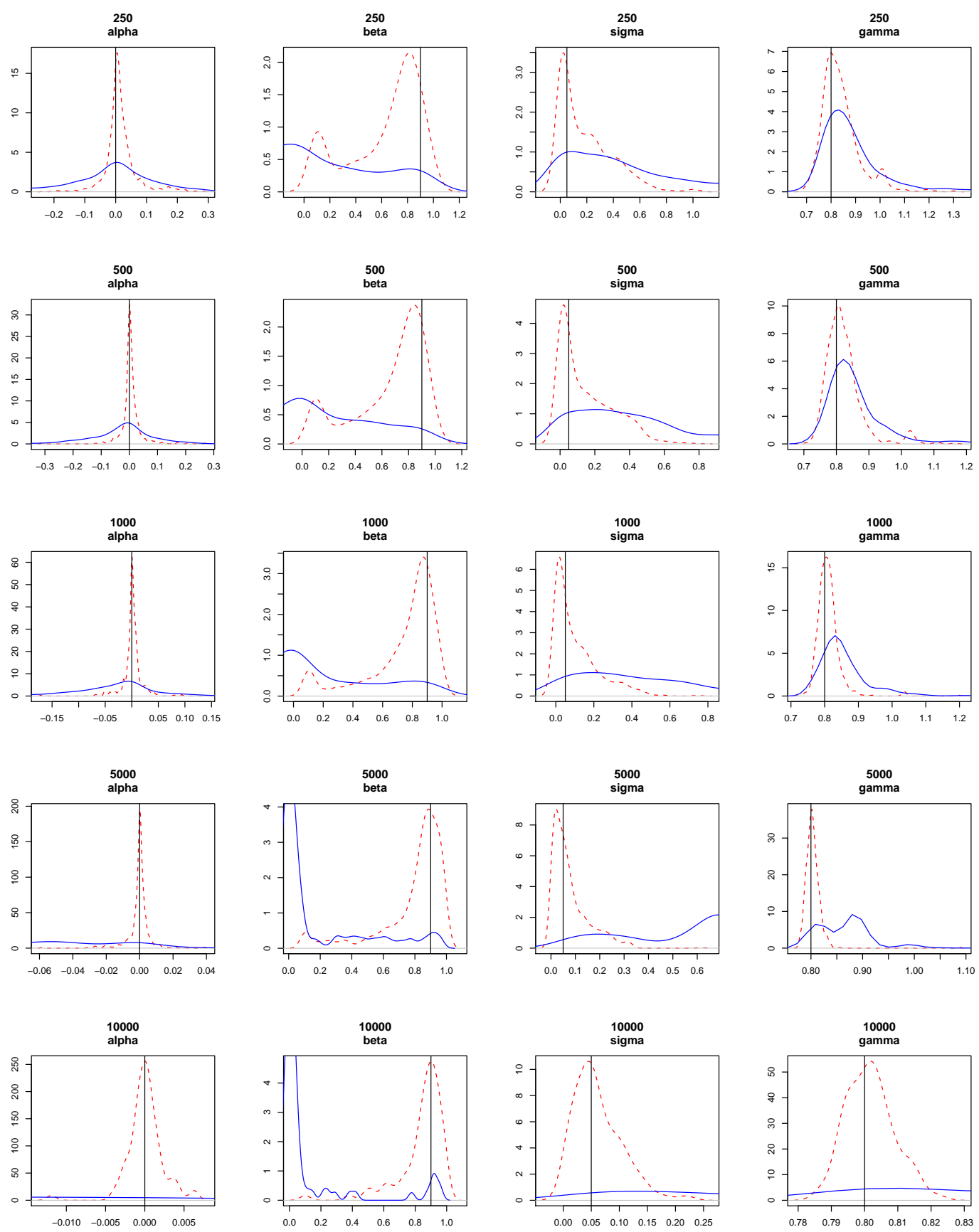

Figure 3: Sampling densities of 1000 EIS-ML (red, dashed) and QML (blue, full) estimates of the parameters of an SCD model with parameters $\omega=0.0, \beta=0.9, \sigma=0.05, \gamma=0.8$. 

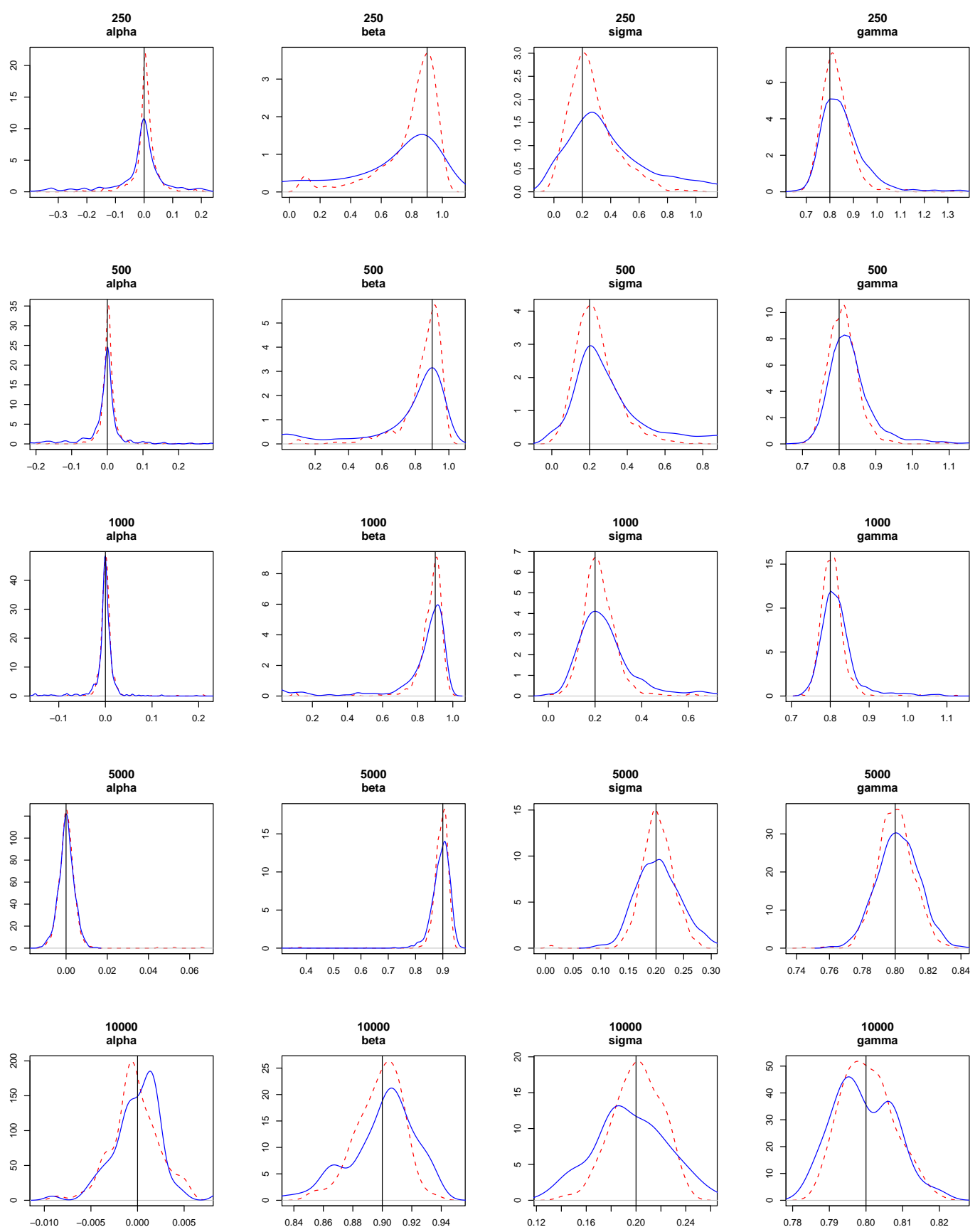

Figure 4: Sampling densities of 1000 EIS-ML (red, dashed) and QML (blue, full) estimates of the parameters of an SCD model with parameters $\omega=0.0, \beta=0.9, \sigma=0.2, \gamma=0.8$. 

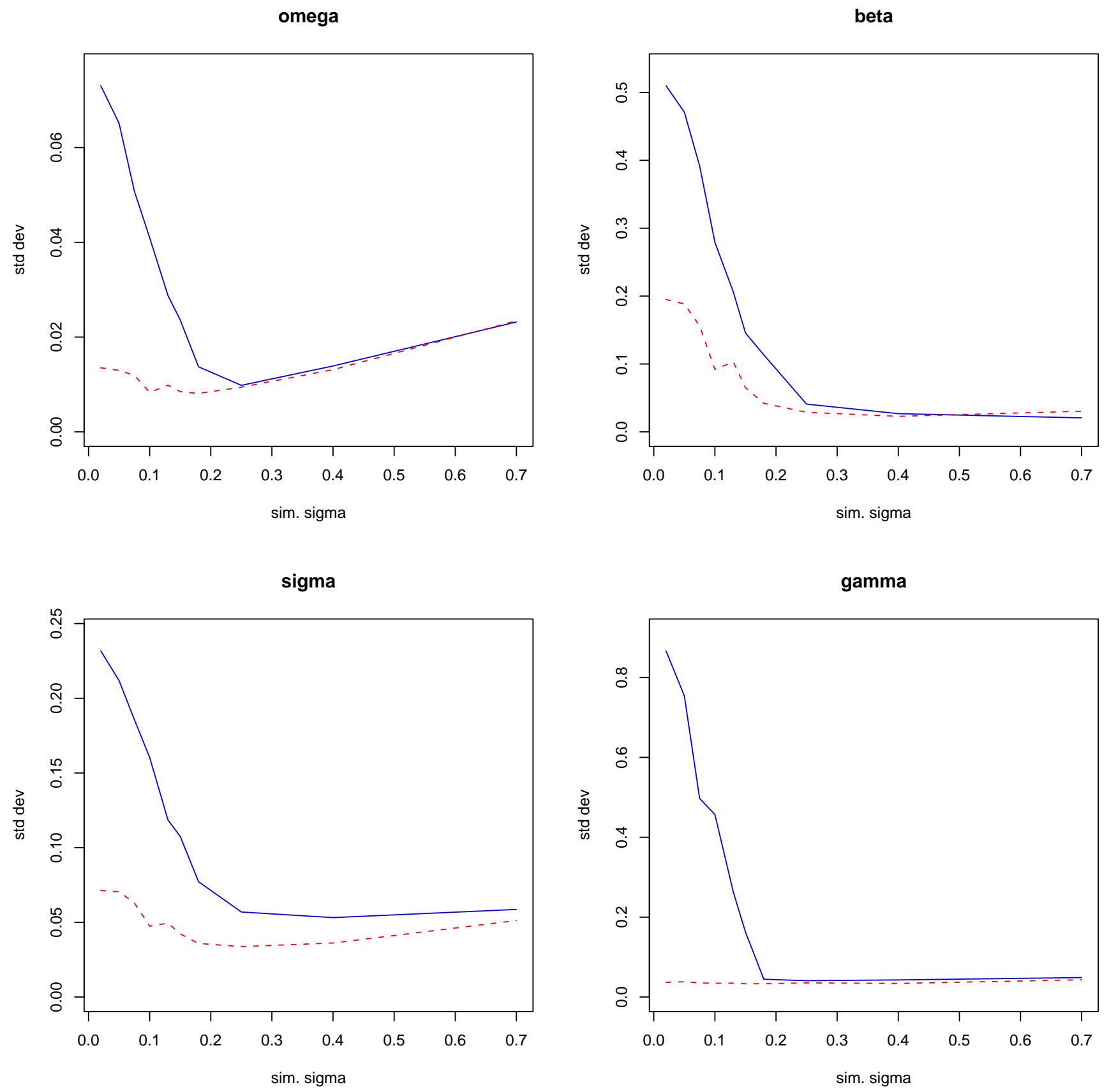

Figure 5: Standard errors of 1000 estimates of the SCD model parameters as a function of the DGP value of $\sigma$. Case of 1000 observations simulated from the DGP with parameters $\omega=0.0, \beta=0.9, \gamma=1.1$. 
Département des Sciences Économiques de l'Université catholique de Louvain

Institut de Recherches Économiques et Sociales

Place Montesquieu, 3

1348 Louvain-la-Neuve, Belgique 